\title{
Richtlinien zur Behandlung des Status epilepticus*
}

\author{
Vorgestellt im Workshop «Status epilepticus» im Rahmen der 173. Tagung der Schweizerischen Neurologischen Gesellschaft, \\ Luzern, 13. Mai 2004
}

D. Leppert, H. R. Stöckli, P. Fuhr, für die Status-epilepticus-Konsensuskonferenz der Nordwestschweizer Spitäler ${ }^{a}$

\section{Zusammenfassung}

1. Der Status epilepticus ist ein Notfall, der unbehandelt zu bleibender neuronaler Schädigung und bei 30-50\% der Patienten zum Tod führt. Er ist definiert als ein über 5 Minuten anhaltender epileptischer Anfall oder eine Abfolge von epileptischen Anfällen, zwischen denen keine vollständige Erholung der Hirnfunktion eintritt. Bei 30-50\% der Patienten tritt der Status epilepticus ohne vorbestehende Epilepsie auf. Die frühzeitige Behandlung kann in den meisten Fällen den Status unterbrechen und führt zu einer vollständigen klinischen Restitution.

2. Pathogenetisch liegt dem Status eine anhaltende oder repetitive, nicht selbst-limitierte elektrische Hyperaktivität von zerebralen Neuronen zugrunde. Diese epileptische Aktivität kann fokal oder generalisiert auftreten.

3. Phänomenologisch manifestiert sich der Status meist mit unwillkürlicher generalisierter oder fokaler motorischer Hyperaktivität (z. B. vom tonisch-klonischen Typ). 30-40\% der Statuspatienten weisen jedoch keine oder nur minimale motorische Phänomene auf und werden der Gruppe des nicht-konvulsiven Status epilepticus zugeteilt.

4. Die Notfalldiagnostik umfasst Analysen zum Nachweis bzw. Ausschluss statusauslösender metabolischer Störungen (z.B. Hypoglykämie und Elektrolytstörungen), Intoxikationen (Alkohol) und fehldosierter Medikamente, speziell von Antiepileptika. In der Notfallsituation ist ein EEG bei einem (aufgrund der motorischen Phänomeme) klinisch offensichtlichen Status epilepticus bedeutungslos, verzögert aber den Behandlungsbeginn. Hingegen ist das EEG angezeigt zur Diagnose eines nicht-konvulsiven Status epilepticus.

Korrespondenz:
Prof. Dr. med. David Leppert

Abteilung für Klinische

Neurophysiologie

Neurologische Klinik

Universitätsspital Basel

CH-4031 Basel

E-Mail: David.X.Leppert@gsk.com weisen Eskalation einer intravenösen Therapie mit dem Ziel, die epileptische Aktivität anhaltend zu unterbrechen:
Stufe 1 (Prähospitalisationsphase): mit einem Benzodiazepin, am besten geeignet ist Lorazepam $^{\mathrm{b}}$ (4 mg in 2 Min.). Mit einem Benzodiazepin iv gelingt es, in $80 \%$ den Status zu unterbrechen.

Stufe 2 (Hospitalisation): Phenytoin ist die bewährte, Valproat ${ }^{\text {b }}$ eine wahrscheinlich gleich wirksame Statusbehandlung (die in der Schweiz noch nicht offiziell zugelassen ist). Die beiden Medikamente unterscheiden sich jedoch hinsichtlich Indikationen (Phenytoin unwirksam bzw. schädlich im Absenzenstatus) und vor allem auch hinsichtlich Nebenwirkungen (Valproat ist kontraindiziert bei schwerer Hepatopathie und Blutungsneigung; Phenytoin kann Herzrhythmusstörungen und Blutdruckabfälle verursachen).

Stufe 3 (Anästhesie): Midazolam und neuerdings Propofol (in Kombination mit einem Benzodiazepin) sind die Alternativen. Bei anhaltendem Status kommt als letzte Möglichkeit die Barbiturat-Narkose zum Einsatz.

\section{Hinweise}

* Ratifiziert von der Chefärztekonferenz der Schweizerischen Neurologischen Gesellschaft. Die Übersetzung des Textes wurde durch einen Beitrag der Firmen Desitin und UCB finanziert. Der Volltext dieser Behandlungsrichtlinien kann in deutsch unter www.saez.ch/status_epilepticus _d.pdf abgerufen werden.

a Dr. H. Brunnschweiler, REHA-Klinik Rheinfelden; Prof. Dr. J. Drewe, Pharmakologie; Prof. Dr. P. Fuhr, Klin. Neurophysiologie, Kantonsspital Basel; Dr. C. Gobbi, Klin. Neurophysiologie, Universitätsspital Basel; Dr. P. Hilti, Medizin, Universitätsspital Basel; Prof. Dr. D. Leppert, Klin. Neurophysiologie, Universitätsspital Basel; Dr. R. Lötscher, Kantonsspital Liestal; Prof. Dr. J. Lütschg, UKBB; Prof. Dr. O. Meienberg, Praxis Binningen; PD Dr. H. Pargger, Anästhesie, Universitätsspital Basel; Dr. D. Schultze, Kantonsspital Bruderholz; Dr. H. R. Stöckli, Kantonsspital und Praxis Liestal.

Externe Mitarbeiter: Prof. P. A. Despland Neurologie, CHUV; Dr. T. Dorn, Dr. G. Krämer, 
Schweizerisches Epilepsiezentrum Zürich; Dr. C. Lienert, Neurologische Universitätsklinik, Inselspital Bern; Prof. H. G. Wieser, PD Dr. A. Siegel, Neurologische Klinik, Universitätsspital Zürich; PD Dr. M. Seeck, PD Dr. B. Walder, Hôpital Universitaire de Genève; Prof. U. Honegger, Pharmakologisches Institut, Universität Bern; PD Dr. B. Tettenborn, Kantonsspital St. Gallen; Prof. U. W. Buettner, Dr. H. Hungerbühler, Dr. M. Tröger, Kantonsspital Aarau. b Die Autoren machen aus formellen Gründen darauf aufmerksam, dass Lorazepam (Temesta ${ }^{\circledR}$ ) für die Behandlung des Status epilepticus in der Schweiz, anders als in Nordamerika und den Ländern der Europäischen Union, nicht zugelassen ist. Die Schweizerische Neurologische Gesellschaft wird sich zusammen mit der betreffenden Pharmafirma um die Zulassung bemühen. Valproat ist derzeit nur in Norwegen für den Status epilepticus zugelassen. Ein Antrag an die Swissmedic zur Revision eines ablehnenden Entscheides wurde durch Prof. H. G. Wieser eingereicht.

\title{
Directives pour le traitement de l'état de mal épileptique*
}

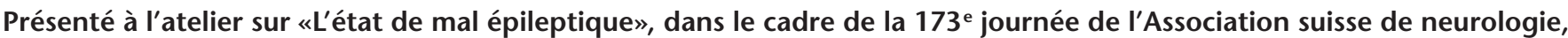 \\ Lucerne, le 13 mai 2004
}

D. Leppert, H. R. Stöckli, P. Fuhr, pour la conférence de consensus sur l'état de mal épileptique des hôpitaux du Nord-Ouest suissea

Correspondance:

Prof. Dr David Leppert Division de neurophysiologie clinique

Clinique de neurologie Hôpital universitaire de Bâle CH-4031 Bâle

\section{Résumé}

1. L'état de mal épileptique (EME) est un cas d'urgence qui, non traité, peut entraîner un dommage neuronal irréversible et conduire à la mort du patient dans 30 à 50\% des cas. Il est défini comme une crise d'épilepsie durant plus de 5 minutes, ou une succession de crises épileptiques entre lesquelles il n'y a pas eu de récupération complète de la fonction cérébrale. Le traitement précoce parvient, dans la plupart des cas, à interrompre l'EME et conduit à une complète restitution clinique.

2. Sur le plan de la pathogénèse, l'EME a pour origine une hyperactivité électrique non autolimitée, persistante ou répétitive des neurones cérébraux. Cette activité épileptique peut survenir de manière focalisée ou généralisée.

3. Sur le plan phénoménologique, l'EME se manifeste le plus souvent par une hyperactivité involontaire généralisée, ou motrice focale (p.ex. de type tonico-clonique). 30 à $40 \%$ des patients souffrant d'un EME ne montrent cependant pas de phénomènes moteurs ou seulement dans une envergure minimale. Ils sont intégrés dans le groupe des EME non convulsifs (EMENC).

4. Le diagnostic d'urgence comprend des analyses permettant de révéler la présence ou l'ab- sence de troubles métaboliques déclencheurs de l'EME (p.ex. l'hypoglycémie et les troubles électrolytiques), les intoxications (alcool) et les médicaments mal dosés, en particulier les antiépileptiques. En situation d'urgence, un EEG est sans signification lors d'un EME cliniquement évident (en raison des phénomènes moteurs). Il ne fait que retarder le début du traitement. En revanche, l'EEG est indiqué pour le diagnostic d'un état de mal épileptique non convulsif.

5. Le traitement a lieu sous forme d'une thérapie intraveineuse, intensifiée progressivement en vue de stopper durablement l'activité épileptique.

Niveau 1 (phase de préhospitalisation): avec benzodiazépine, le lorazépam ${ }^{\text {b }}$ est le mieux indiqué (4 mg en $2 \mathrm{~min}$ ). Avec une benzodiazépine i.v., l'état de mal est interrompu dans $80 \%$ des cas.

Niveau 2 (hospitalisation): la phénytoïne est le médicament de choix, le valproate ${ }^{\mathrm{b}}$ est un traitement probablement tout aussi efficace (pas encore officiellement admis par la Suisse). Les deux médicaments se différencient néanmoins par leurs indications (la phénytoïne est inefficace, ou même dangereuse en cas d'état d'absence) et surtout par leurs effets secondaires (le valproate est contre-indiqué 
en cas d'hépatopathie grave et de tendance aux saignements; la phénytoïne peut provoquer des troubles du rythme cardiaque et des chutes de tension).

Niveau 3 (anesthésie): le midazolam et, c'est nouveau, le propofol (en combinaison avec une benzodiazépine) sont les alternatives. Lors d'état de mal épileptique prolongé, la dernière possibilité se présente sous la forme d'une anesthésie aux barbituriques.

\section{Indications}

* Ratifiés par la conférence des médecins-chefs de l'Association suisse de neurologie. La traduction du texte est financée par une contribution des firmes Desitin et UCB. La teneur intégrale de ces directives de traitement peut être obtenue à l'adresse suivante: www.saez.ch/status_epilepticus _f.pdf

a Dr H. Brunnschweiler, Clinique de réadaptation (REHA-Klinik), Rheinfelden; Prof. J. Drewe, pharmacologie; Prof. P. Fuhr, Clinique de neurophysiologie, Hôpital universitaire de Bâle; Dr C. Gobbi, Clinique de neurophysiologie, Hôpital universitaire de Bâle; Dr P. Hilti, dépt de médecine, Hôpital universitaire de Bâle; Prof. Dr D. Leppert, Clinique de neurophysiologie, Hôpital universitaire de Bâle; Dr R. Lötscher, Hôpital cantonal de Liestal; Prof. J. Lütschg, UKBB; Prof. O. Meienberg, cabinet médical à Binningen; Dr H. Pargger, dépt d'anesthésie, Hôpital universitaire de Bâle; Dr D. Schultze, Hôpital cantonal de Bruderholz; Dr H. R. Stöckli, Hôpital cantonal et cabinet médical à Liestal.

Collaborateurs externes: Prof. P. A. Despland, dépt de neurologie, CHUV; Dr T. Dorn, Dr G. Krämer, Centre suisse de l'épilepsie, Zurich; Dr C. Lienert, Clinique universitaire de neurologie, Hôpital de l'Ile, Berne: Prof. H.G. Wieser, Dr A. Siegel, p.-d., Clinique neurologique, Hôpital universitaire de Zurich; Dr M. Seeck, p.-d., Dr B. Walder, p.-d., Hôpital universitaire de Genève; Prof. U. Honegger, Institut pharmacologique, Université de Berne; Dr B Tettenborn, p.-d., Hôpital cantonal de SaintGall; Prof. U. W. Buettner, Dr H. Hungerbühler, Dr M. Tröger, Hôpital cantonal d'Aarau.

b Les auteurs tiennent à préciser sur le plan formel que le lorazépam (Temesta $\left.{ }^{\circledR}\right)$, contrairement aux Etats-Unis et aux pays de l'Union européenne, n'est pas admis pour le traitement de l'état de mal épileptique en Suisse. L'Association suisse de neurologie a l'intention de s'efforcer d'obtenir cette admission en prenant contact avec la firme pharmaceutique concernée. Le valproat n'est actuellement admis pour les cas de mal épileptique qu'en Norvège. Le Prof. H. G. Wieser a déposé une demande à Swissmedic pour la révision de la décision négative.

\title{
Direttive per il trattamento dello stato di male epilettico*
}

\author{
Presentato all'atelier «Status epilepticus» nell'ambito della 173esima giornata dell'Associazione svizzera di neurologia, \\ Lucerna, 13 maggio 2004
}

D. Leppert, H. R. Stöckli, P. Fuhr, per la conferenza di consenso sullo stato di male epilettico degli ospedali della Svizzera nordoccidentale ${ }^{a}$

Corrispondenza:

Prof. Dr. med. David Leppert Divisione di neurologia clinica Clinica neurologica

Ospedale universitario di Basilea CH-4031 Basilea

\section{Riassunto}

1. Lo stato di male epilettico è un'urgenza, che se non trattato conduce ad un danno neuronale permanente e può essere letale nel $30-50 \%$ dei casi. Viene definito come una continua attività epilettica di durata superiore ai 5 minuti o come una sequenza di crisi epilettiche tra le quali non vi sia un pieno ristabilimento delle funzioni cerebrali. Nel $30-50 \%$ dei casi si tratta della prima manifestazione epilettica (anamnesi personale negativa per epilessia). Il trattamento precoce può nella maggior parte dei casi interrompere lo stato di male epilettico e condurre ad una risoluzione clinica completa.

2. Dal profilo patogenetico si tratta di una condizione di attività elettrica neuronale permanente o ripetitiva e non autolimitante. L'attività epilettica può essere focale o generalizzata.

3. Dal profilo fenomenologico lo stato di male epilettico si presenta solitamente con movimenti stereotipati involontari focali o generalizzati (ad esempio crisi tonico-clonica). Nel 30-40\% dei casi non si riscontra durante 
lo stato di male epilettico alcuna attività motoria, si tratta in questo caso di uno stato di male epilettico non convulsivo.

4. Gli accertamenti d'urgenza comprendono la ricerca e l'esclusione di fattori metabolici scatenanti uno stato di male epilettico (p.es. ipoglicemia, diselettrolitemia), l'intossicazione (alcool) e/o il dosaggio errato di farmaci, in particolare di antiepilettici. Nella situazione d'urgenza con crisi epilettica motoria manifesta è superfluo eseguire un elettroencefalogramma in quanto questo ritarderebbe unicamente l'inizio della terapia specifica. Per contro un elettroencefalogramma tempestivo è sempre indicato nel sospetto di uno stato di male epilettico non convulsivo.

5. Il principio della terapia si svolge secondo un algoritmo di progressiva escalazione della terapia intravenosa con lo scopo di interrompere l'attività epilettica:

stadio 1 (prima del ricovero): inizio con una benzodiazepina, si consiglia con lorazepam ${ }^{\mathrm{b}}$ (4 mg in $2 \mathrm{~min}$ ). Con una benzodiazepina si può interrompere uno stato di male epilettico ca. nel $80 \%$ dei casi.

stadio 2 (ricovero): fenitoina o acido valproico $^{\mathrm{b}}$. Entrambe le sostanze sono da considerarsi farmaci di prima scelta. Valutazione del singolo farmaco a seconda della situazione.

stadio 3 (anestesia): le alternative sono il midazolam o il propofolo (sempre combinato con una benzodiazepina). Nel caso di uno stato di male epilettico farmaco-resistente considerare l'inizio di una terapia con barbiturati.

\section{Indicazioni}

* Ratificate dalla conferenza dei primari dell'Associazione Svizzera di Neurologia. Le traduzioni del testo sono stati finanziati dalle ditte Desitin e UCB. Il testo integrale di queste direttive di trattamento può essere ottenuto all'indirizzo seguente: www.saez.ch/status_epilepticus_i.pdf.

a Dr. H. Brunnschweiler, Clinica di riabilitazione di Rheinfelden; Prof. Dr. J. Drewe, farmacologia; Prof. Dr. P. Fuhr, Clinica neurofisiologica, Ospedale universitario di Basilea; Dr. C. Gobbi, Clinica neurofisiologica, Ospedale universitario di Basilea; Dr. P. Hilti, dipartimento di medicina, Ospedale universitario di Basilea; Prof. Dr. D. Leppert, Clinica neurofisiologica, Ospedale universitario di Basilea; Dr. R. Lötscher, Ospedale cantonale di Liestal; Prof. Dr. J. Lütschg, UKBB; Prof. Dr. O. Meienberg, studio medico Binningen; PD Dr. H. Pargger, dipartimento di anestesia, Ospedale universitario di Basilea; Dr. D. Schultze, Ospedale cantonale di Bruderholz; Dr. H. R. Stöckli, Ospedale cantonale e studio medico Liestal.

Collaboratori esterni: Prof. P.A. Despland, dipartimento di neurologia, CHUV; Dr. T. Dorn, Dr. G. Krämer, Centro svizzero di epilessia Zurigo; Dr. C. Lienert, Clinica universitaria neurologica, Inselspital Berna; Prof. H. G. Wieser, PD Dr. A. Siegel, Clinica neurologica, Ospedale universitario di Zurigo; PD Dr. M. Seeck, PD Dr. B. Walder, Ospedale universitario di Ginevra; Prof.

U. Honegger, Istituto di farmacologia, Università Berna; PD Dr. B. Tettenborn, Ospedale cantonale di San Gallo; Prof. U. W. Buettner, Dr. H. Hungerbühler, Dr. M. Tröger, Ospedale cantonale di Aarau.

b Per motivi formali gli autori fanno notare che in Svizzera il lorazepam $\left(\right.$ Temesta $\left.^{\circledR}\right)$, contrariamente agli Stati Uniti e ai paesi dell'Unione europea non è ammesso per il trattamento dello stato di male epilettico. L'Associazione svizzera di neurologia ha l'intenzione di impegnarsi per ottenere quest'ammissione prendendo contatto con la società farmacologia interessata. Il valproat è attualmente ammesso per i casi di mal epilettico solamente in Norvegia. Il Prof. H. G. Wieser ha presentato una domanda a Swissmedic per la revisione della decisione negativa. 